REVISTA MATEMÁTICA de la

Universidad Complutense de Madrid

Volumen 9, número 2: 1996

http://dx.doi.org/10.5209/rev_REMA.1996.v9.n2.17588

\title{
A remark on the blow-up of the solutions of the equation $u_{t}+f(x) a(u) u_{x}=h(x, u)$
}

\section{João-Paulo DIAS and Mário FIGUEEIRA}

\begin{abstract}
We consider the Cauchy problem for the equation $u_{t}+$ $f(x) a(u) u_{x}=h(x, u)$ where $f, a$ and $h$ are real $C^{1}$ functions, $f \geq$ $\theta>0, a^{\prime}>0, h_{u} \geq 0$ and $h_{x} \leq 0$. Following the ideas of Lax [4] and Klainerman-Majda [3], we prove a blow-up result for the solutions with special data corresponding, in certain cases, to the development of a singularity in $u_{x}$.
\end{abstract}

\section{Introduction and statement of the result}

Let us consider the scalar conservation law

$$
u_{t}+a(u) u_{x}=0, \quad a \in C^{1}(\mathbf{R}),(x, t) \in \mathbf{R}^{2}
$$

The study of the development of singularities for the solution of the Cauchy problem for the equation (1.1) has been treated by Lax [4] and Majda [6] by proving the appearence of shocks if we impose some conditions to the function $a$ and to the initial data $u_{0}$. In this paper we extend some of these results to the equation

$$
u_{t}+f(x) a(u) u_{x}=h(x, u), \quad f, a \in C^{1}(\mathbf{R}), h \in C^{1}\left(\mathbf{R}^{2}\right)
$$

by using a method similar to the one employed by Klainerman - Majda [3] for a system of conservation laws.

1991 Mathematics Subject Classification: 35L65, 35L67

Servicio Publicaciones Univ. Complutense. Madrid, 1996. 
For the special cases of the equation (1.2) related to the generalised Burgers equation, Natalini - Tesei [8] gave some conditions for the initial data in order to obtain blow-up results for the $L^{\infty}$ norm of the solution. In the last section we give some applications to a class of equations arising in physics.

We assume

$$
\begin{aligned}
& f(\xi) \geq \theta>0, \quad a^{\prime}(\xi) \geq \rho>0, \forall \xi \in \mathbf{R}, \quad h_{u} \geq 0, h_{x} \leq 0 \quad(1.3) \\
& \text { and } f \in W^{1, \infty}(\mathbf{R}), \quad h(., \xi) \in W^{1, \infty}(\mathbf{R}) \quad \text { for each } \xi \in \mathbf{R} .
\end{aligned}
$$

Following Douglis [1] and Li-Yu [5], ch.1, if we take the initial data $u_{0} \in C^{1}(\mathbf{R}) \cap W^{1, \infty}(\mathbf{R})$ then there exists a unique local solution

$$
u \in C^{1}\left(\mathbf{R} \times\left[0, T_{0}\right]\right) \cap C^{1}\left(\left[0, T_{0}\right] ; L^{\infty}(\mathbf{R})\right) \cap C\left(\left[0, T_{0}\right] ; W^{1, \infty}(\mathbf{R})\right)
$$

of the equation (1.2) such that $u(\alpha, 0)=u_{0}(\alpha), \forall \alpha \in \mathbf{R}$. We will denote by $\left[0, T^{\prime}\right]$ the corresponding maximal interval of existence where the sharp continuation principle (cf. [6], 2.3), can be applied.

For such a solution let us consider the equation of the characteristics

$$
\frac{d x}{d t}(t)=f(x(t)) a(u(x(t), t)) \quad \text { with } \quad x(0)=\alpha, \alpha \in \mathbf{R} .
$$

Along this ,characteristic curve the solution $u$ satisfies the differential equation

$$
\frac{d}{d t} u(x(t), t)=h(x(t), u(x(t), t)) \quad \text { with } \quad u(x(0), 0)=u(\alpha, 0)=u_{0}(\alpha)
$$

We can now state our result which extends previous results of Lax [4] and Majda [6] for conservation laws.

Theorem 1 Under the above assumption (1.3) consider the unique local solution $u$ of (1.2) for the initial data $u_{0} \in C^{1}(\mathbf{R}) \cap W^{1, \infty}(\mathbf{R})$ and assume that $u \in L^{\infty}\left(\mathbf{R} \times\left[0, T^{\prime}[)\right.\right.$ where $\left[0, T^{\prime}[\right.$ is the corresponding maximal interval of existence. Let $\alpha_{0} \in \mathbf{R}$ be such that $u_{0}^{\prime}\left(\alpha_{0}\right)<0$ and let $x(t)=$ $x\left(t ; \alpha_{0}\right)$ be the corresponding characteristic curve starting in $x(0)=\alpha_{0}$. Then, or $\limsup _{t \rightarrow T^{\prime}}\left(\left\|u_{x}(., t)\right\|_{L^{\infty}}+\left\|u_{t}(., t)\right\|_{L^{\infty}}\right)=+\infty$

$$
\text { or } \quad \liminf _{t \rightarrow T^{\prime}} \int_{0}^{t} a^{\prime}(u(x(\tau), \tau)) u_{x}(x(\tau), \tau) d \tau=-\infty
$$


and hence $\liminf _{t \rightarrow T^{\prime}} u_{x}(x(t), t)=-\infty$.

Moreover $T^{\prime} \leq T^{*}=\left(-\rho f\left(\alpha_{0}\right) u_{0}^{\prime}\left(\alpha_{0}\right)\right)^{-1}$.

Remark. Since we suppose $u \in L^{\infty}\left(\mathbf{R} \times\left[0, T^{\prime}[)\right.\right.$, it is enough to assume $a^{\prime}>0$ to prove the blow-up result.

The authors are indebted to Prof. L. Sanchez for stimulating discussions and to Prof. Li Ta-tsien who has pointed out a mistake in a first redaction of this note and suggested some other improvements.

This research was partially supported by the European Project HCM № CHRX/CT93/0407.

\section{Proof of Theorem 1.}

Following an idea of Klainerman - Majda [3] we have, along the characteristic curve defined by (1.5),

$$
\frac{d}{d t}\left(\frac{\partial x}{\partial \alpha}\right)=\frac{\partial}{\partial \alpha}\left(\frac{d x}{d t}\right)=f^{\prime} \frac{\partial x}{\partial \alpha} a(u)+f a^{\prime}(u) \frac{\partial u}{\partial \alpha}
$$

Hence, by the theory of linear ordinary differential equations, we have

$$
\frac{\partial x}{\partial \alpha}(t)=\left(\exp \int_{0}^{t} f^{\prime} a(u) d \tau\right)\left\{1+\int_{0}^{t} f a^{\prime}(u) \frac{\partial u}{\partial \alpha}\left[\exp \left(-\int_{0}^{s} f^{\prime} a(u) d \tau\right)\right] d s\right\}
$$

On the other hand, by the results on the derivative of the solution of an ordinary differential equation in order to the initial data (cf. Petrovski [9], for example) we obtain, from (1.5),

$$
\frac{\partial x}{\partial \alpha}(t ; \alpha)=\exp \int_{0}^{t} \frac{\partial}{\partial x}(f a(u))(x(\tau), \tau) d \tau
$$

Also, we have, from (1.6), if $u_{0}^{\prime}(\alpha) \neq 0$,

$$
\frac{d}{d t}\left(\frac{\partial u}{\partial u_{0}}\right)=h_{u} \frac{\partial u}{\partial u_{0}}+h_{x} \frac{\partial x}{\partial u_{0}}=h_{u} \frac{\partial u}{\partial u_{0}}+h_{x} \frac{\partial x}{\partial \alpha}\left(u_{0}^{\prime}(\alpha)\right)^{-1}
$$

Hence, in a neighborhood of $\alpha_{0} \in \mathbf{R}$ such that $u_{0}^{\prime}\left(\alpha_{0}\right)<0$ we have (since $h_{u} \geq 0, h_{x} \leq 0$ and $\frac{\partial x}{\partial \alpha} \geq 0$ ), by (2.3),

$$
\frac{\partial u}{\partial u_{0}}\left(t ; u_{0}\right) \geq \frac{\partial u}{\partial u_{0}}\left(0 ; u_{0}\right)=1
$$


and so

$$
\frac{\partial u}{\partial \alpha}(t ; \alpha)=\frac{\partial u}{\partial u_{0}}\left(t ; u_{0}\right) u_{0}^{\prime}(\alpha) \leq u_{0}^{\prime}(\alpha)<0
$$

Furthermore, since $f>0$, we obtain from (1.5), along the characteristic curve

$$
f^{\prime} a(u)=\frac{f^{\prime}}{f} \frac{d x}{d t}=\frac{d}{d t} \log f
$$

and so

$$
\exp \left(\int_{0}^{t} f^{\prime} a(u) d \tau\right)=\frac{f(x(t))}{f(\alpha)}
$$

Introducing (2.4) and (2.5) in (2.1) we obtain

$$
\frac{\partial x}{\partial \alpha}(t ; \alpha)=\frac{f(x(t))}{f(\alpha)}\left[1+f(\alpha) \int_{0}^{t} a^{\prime}(u(x(s), s)) \frac{\partial u}{\partial u_{0}}\left(s ; u_{0}\right) u_{0}^{\prime}(\alpha) d s\right]
$$

Hence, since by (1.3) $a^{\prime} \geq \rho>0$. we obtain if $u_{0}^{\prime}\left(\alpha_{0}\right)<0$, by applying (2.4) and (2.6),

$$
\frac{\partial x}{\partial \alpha}\left(t ; \alpha_{0}\right) \leq \frac{f(x(t))}{f\left(\alpha_{0}\right)^{-}}\left(1+\rho f\left(\alpha_{0}\right) u_{0}^{\prime}\left(\alpha_{0}\right) t\right)
$$

and the right hand side is equal to zero for $t=T^{*}=\left(-\rho f \cdot\left(\alpha_{0}\right) u_{0}^{\prime}\left(\alpha_{0}\right)\right)^{-1}$. Introducing (2.5) in (2.2) we derive

$$
\frac{\partial x}{\partial \alpha}(t ; \alpha)=\frac{f(x(t))}{f(\alpha)} \exp \left(\int_{0}^{t}\left(f a^{\prime}(u) u_{x}\right)(x(\tau), \tau) d \tau\right)
$$

and so, by (2.7) and (2.8), there exists a $T \leq T^{*}$ such that, or

$$
\underset{t \rightarrow T^{\prime}}{\limsup }\left(\left\|u_{x}(., t)\right\|_{L^{\infty}}+\left\|u_{t}(., t)\right\|_{L^{\infty}}\right)=+\infty
$$

or

$\liminf _{t \rightarrow T} \frac{\partial x}{\partial \alpha}\left(t ; \alpha_{0}\right)=\liminf _{t \rightarrow T} \frac{f(x(t))}{f\left(\alpha_{0}\right)} \exp \left(\int_{0}^{t}\left(f a^{\prime}(u) u_{x}\right)(x(\tau), \tau) d \tau\right)=0$,

that is, since $f(x) \geq \theta>0$,

$$
\liminf _{t \rightarrow T} \int_{0}^{t}\left(a^{\prime}(u) u_{x}\right)(x(\tau), \tau) d \tau=-\infty
$$


and the theorem is proved.

\section{Examples.}

Our first example of application of theorem 1 is a semi-linear perturbation of the Burgers equation which can not be reduced by a suitable transformation to the Burgers equation in the framework of [2]:

$$
u_{t}+u u_{x}=\lambda u^{p}, \text { for odd } p>1 \text { and } \lambda>0
$$

For this equation we obtain the following blow-up result under the assumptions of theorem 1 :

$$
\liminf _{t \rightarrow T} \int_{0}^{t} u_{x}(x(\tau), \tau) d \tau=-\infty
$$

for a certain $T \leq T^{*}=\left(-u_{0}^{\prime}\left(\alpha_{0}\right)\right)^{-1}$ if $u_{0}^{\prime}\left(\alpha_{0}\right)<0$ and where $x(t)$ is the characteristic curve corresponding to $x(0)=\alpha_{0}$.

Other kind of results, concerning the blow-up of the $L^{\infty}$ space norm of the solutions of (3.1) for suitable initial data can be found in [8].

Now, consider the more general equation

$$
u_{t}+a(u) u_{x}+\lambda h(u)=0,
$$

with $\lambda<0, a^{\prime}(\xi) \geq \rho>0, \forall \xi \in \mathbf{R}$ and $h^{\prime} \geq 0$. These equations are introduced in [7] (with the suplementary condition $h^{\prime}(\xi)>0$ for $\xi>0$ ) and appear in the study of the so called Gunn effect in semiconductors. The situation described in theorem 1 corresponds to the appearence of shocks pointed out in section 2.4 of [7] in the case of the existence of a negative dissipation term.

Finally, consider the equation (3.2) in the special case

$$
u_{t}+u^{k} u_{x}-u^{p}=0, \quad 0<p<1, k>0,
$$

for positive solutions (see [7] for the positive dissipation case). For this equation the theorem 1 can not be applied without modification. Take a smooth strictly positive initial data $u_{0}$. Along the characteristic curve $x(t ; \alpha)$ defined by $(1.5)$ we easily obtain

$$
u(x(t ; \alpha), t)=\left[(1-p) t+u_{0}(\alpha)^{1-p}\right]^{\frac{1}{1-p}}, t \geq 0 .
$$


Following the proof of theorem 1 we derive

$$
\frac{\partial x}{\partial \alpha}(t ; \alpha)=1+k u_{0}^{\prime}(\alpha) u_{0}^{-p}(\alpha) \int_{0}^{t}\left[(1-p) s+u_{0}(\alpha)^{1-p}\right]^{q} d s
$$

where $q=\frac{k-1+p}{1-p}>-1$.

Hence, for $\alpha_{0}$ such that $u_{0}^{\prime}\left(\alpha_{0}\right)<0$, the right hand side of (3.4) attains zero for a certain $T^{*}<+\infty$. Therefore we obtain, as in the proof of theorem 1,

$$
\liminf _{t \rightarrow T^{*}} \int_{0}^{t}\left(u^{k-1} u_{x}\right)\left(x\left(\tau ; \alpha_{0}\right), \tau\right) d \tau=-\infty
$$

and hence $\liminf _{t \rightarrow T^{*}} u_{x}\left(x\left(t ; \alpha_{0}\right), t\right)=-\infty$.

\section{References}

[1] A. Douglis, Some existence theorems for hyperbolic systems of partial differential equations in two independent variables, Comm. Pure Appl. Math., 5, 119-154 (1952).

[2] K.T.Joseph and P.L.Sachdev, On the solution of the equation $u_{t}+u^{n} u_{x}+H(x, t, u)=0$, Quart. of Appl. Math. 52, 519-527 (1994).

[3] S.Klainerman and A.Majda, Formation of singularities for wave equations including the nonlinear vibrating string, Comm. Pure Appl. Math. 33, 241-263 (1980).

[4] P.D.Lax, Development of singularities of solutions of nonlinear hyperbolic partial differential equations, J. Math. Phys. 5, 611-613 (1964).

[5] Li Ta-tsien and Yu Wen-ci, Boundary value problems for quasilinear hyperbolic systems, Duke University Mathematics Series, Vol.5, 1985.

[6] A.Majda, Compressible fluid flow and systems of conservation laws in several space variables, Applied Math. Sciences, Vol. 53, Springer, 1984.

[7] J.D.Murray, On the Gunn effect and other physical examples of perturbed conservation equations, J. Fluid Mech. 44, 315-346 (1970). 
A remark on the blow-up of the solutions of the equations...

[8] R.Natalini and A.Tesei, Blow-up of solutions for a class of balance laws, Comm. Part. Diff. Eq., 19, 417-453 (1994).

[9] I.G.Petrovski, Ordinary differential equations, Prentice-Hall, 1966.

CMAF/University of Lisbon, Recibido: 23 de Octubre de 1995 2 Av. Prof. Gama Pinto, 1699 Lisboa Codex-PORTUGAL 